\title{
On the Electrical Conductivity of the Upper Atmosphere
}

\author{
By Ken-ichi MAEDA
}

Electric Communication Laboratory

\begin{abstract}
The paper dẹals with the problem of the electrical condnctivity supported by the upper atmospheric ionization, to which the diurnal variation of terrestrial magnetism is attributed in the dynamo theory. Number densities of free electrons and ions and their collision frequencies are substantially important for the evaluation of the vertical distribution and integrated value of the electrical conductivity. For this purpose various elementary processes governing the ionospheric characteristics, that is, recombination, attachment, detachment and collision, are discussed on the basis of ionospheric observation with the aid of some theoretical data by other scientists. Although our present knowledges had to face some contradictions on the way of numerical estimation of necessary elements, it is indicated that the total conductivity will be about $2 \times 10^{-7}$ e.m.u. during the dạtytime, with the maximum conductivity of $(1.5 \sim 2.0) \times 10^{-14}$ e.m.u. in the $E$-region.
\end{abstract}

\section{Introduction}

Although many important points on the physical relations between the ionosphere and the terrestrial magnetism have been found up to this time, there still remains the problem of the electrical conductivity of the ionosphere, which has a significant bearing to the physical nature of diurnal variation of the terrestrial magnetism. From the analysis of diurnal variations observed in the terrestrial magnetism it was shown that the total conductivity of the upper atmosphere should be of the order of $10^{-6}$ e.m.u. Recently, however, T. Nagata and others ${ }^{(1)}$ reported the results of study on ionospheric induction effect, and according to their conclusions it will be reasonable to accept that the total conductivity will be less than $10^{-7}$ e.m.u. Sir Edward Appleton? ${ }^{(2)}$ gave a formula with a table to evaluate the total conductivity based on the recombination theory and we can calculate the total conductivity by assuming collision frequencies, number densities of electrons and ions and scale height. As to, the evaluation of density ratio of electron to ion, which is very important to our present problem, we can refer to the reports by G. Goubau, ${ }^{(3)}$ F.L. Mohler, ${ }^{(4)}$ D.R. Bates and H.S.W. Massey ${ }^{(5)}$ and others, 
Helght ( $\mathrm{km}$ )

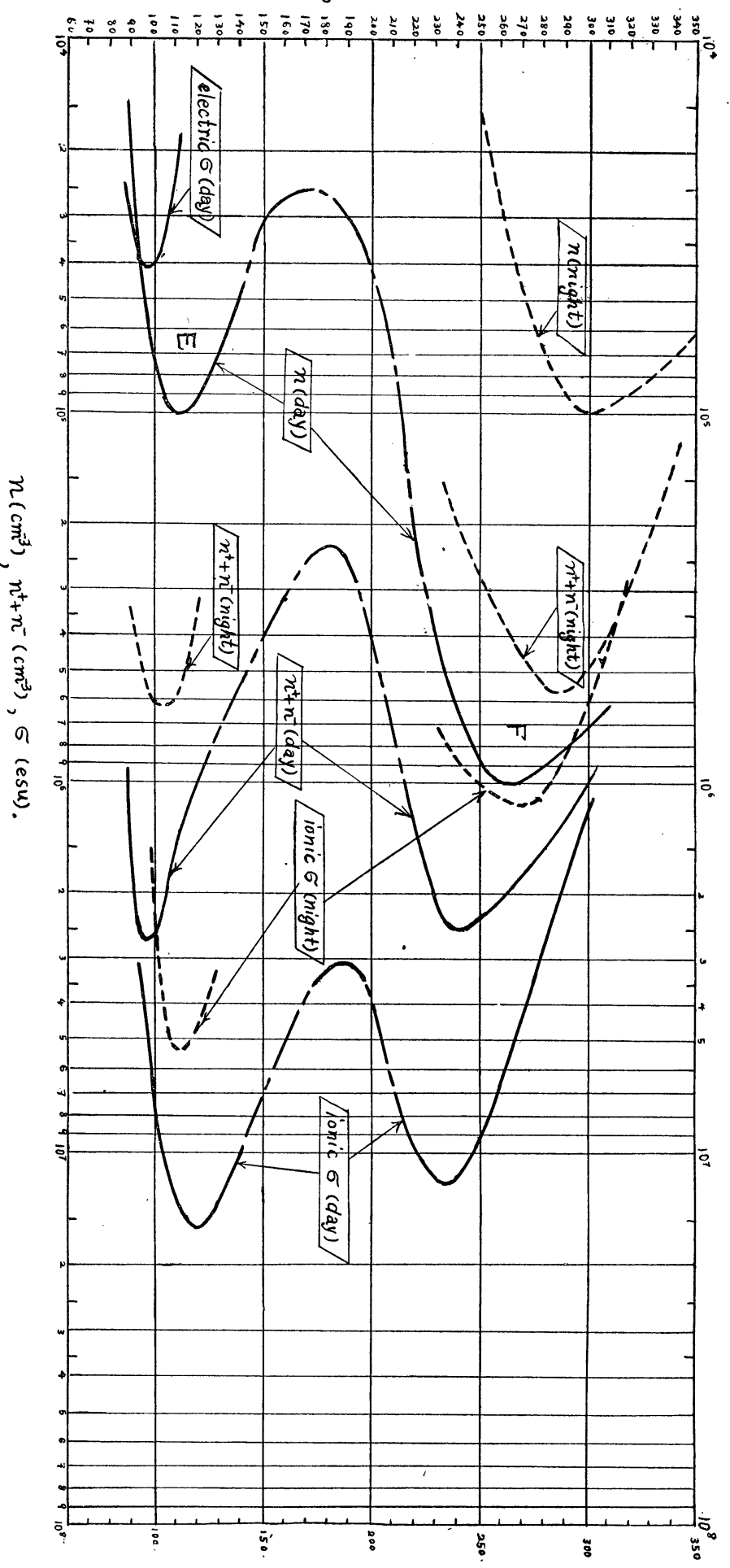

The writer discusses here some problems necessary to find out the density ratio of electron to ion and collision frequencies on the basis of ionospheric measurements together with some theories of other scientists, and the vertical distribution and the integrated value of the electrical conductivity and their changes from day to night are presented.

\section{Mean collision frequencies}

1. Theoretical valves

By theoretical calculaion of T. Yamanouchi ${ }^{(6)}$ the mean collision frequency $(\nu)$ of free electron in $F_{2}$-layer, where Oxygen atom is the controling particle, is given as

$$
\nu \simeq 3 \times 10^{2} \mathrm{sec}^{-1}(\mathrm{~h}=250 \mathrm{~km}) .
$$

In this case the number density of Oxygen atoms is assumed to be about $6 \times 10^{9} /$ c.c. and the temperature as about $1000^{\circ} \mathrm{K}$.

\section{Observed values}

According to the experiments by Farmer, Ratcliffe and others ${ }^{(7)}$ we have the values for electron collision as follows.

$$
\begin{aligned}
& \nu \simeq 10^{5} \mathrm{sec}^{-1} \\
& \quad \text { for } E \text {-layer }(100 \mathrm{~km}), \\
& \nu \simeq 1.5 \times 10^{3} \mathrm{sec}^{-1} \\
& \quad \text { for } F_{2} \text {-layer }(250 \mathrm{~km}) .
\end{aligned}
$$


As to the $F_{2}$-layer we consider the contribution of electronion collision according to $\mathrm{T}$. Yonezawa ${ }^{(s)}$ and adopt the value of $\nu$ a little less than the above value, that is, $(3 \sim 10) \times 10^{2}$ for $F_{2}$-region.

\section{Height dependence}

Height dependence of $\nu$ for electron collision is given by the following relation, ${ }^{(6)}$

$$
\nu \sim N \sqrt{T} \text {, }
$$

where $N$ is the number density of neutral particles and $T$ the electron temperature. For the purpose we have to know the vertical distributions of $N$ and $T$, but it is very difficult at present stage to show them exactly. The following table is presented to show the values of $\nu$ for each layer, together with other various values, with which we consider the problems as our model ionosphere.

Table of Model Ionosphere

\begin{tabular}{|c|c|c|c|c|c|c|c|c|}
\hline Region & $\begin{array}{r}\text { Height } \\
(\mathrm{km})\end{array}$ & Gas & $\begin{array}{c}\text { Tem- } \\
\text { perature } \\
\text { (deg. } \\
\text { Kelvin) }\end{array}$ & $\begin{array}{l}\text { Pressure } \\
\qquad(\mathrm{mmHg})\end{array}$ & $\begin{array}{r}\text { Gas density } \\
\mathrm{N}\left(\mathrm{cm}^{-3}\right)\end{array}$ & $\begin{array}{c}\text { Mean } \\
\text { collision } \\
\text { frequency } \\
\quad \vee\left(\sec ^{-1}\right)\end{array}$ & $\begin{array}{c}\begin{array}{c}\text { Attachment } \\
\text { coefficient }\end{array} \\
\beta\left(\mathrm{sec}^{-1}\right)\end{array}$ & $\begin{array}{l}\text { Apparent re- } \\
\text { combination } \\
\text { coefficient } \\
\alpha^{\prime}\left(\mathrm{cm}^{-3} \mathrm{sec}^{-1}\right)\end{array}$ \\
\hline \multirow{4}{*}{$E$} & \multirow{4}{*}{$\begin{array}{l}110 \\
120\end{array}$} & \multirow{4}{*}{$\mathrm{O}_{2} \& \mathrm{O}$} & \multirow{4}{*}{400} & $2 \times 10^{-3}$ & $1.4 \times 10^{14}$ & $4 \times 10^{5}$ & $7 \times 10^{-2}$ & \multirow{4}{*}{$10^{-8}$} \\
\hline & & & & $5 \times 10^{-4}$ & $3 \times 10^{13}$ & $1 \times 10^{5}$ & $2 \times 10^{-2}$ & \\
\hline & & & & $2 \times 10^{-4}$ & $1 \times 10^{13}$ & $4 \times 10^{4}$ & $8 \times 10^{-3}$ & \\
\hline & & & & $1 \times 10^{-4}$ & $5 \times 10^{12}$ & $2.3 \times 10^{-4}$ & $5 \times 10^{-3}$ & \\
\hline & 200 & & & $\begin{array}{c}2.8 \times 10^{-6} \\
\left(1.6 \times 10^{-5}\right)^{2}\end{array}$ & $\begin{array}{c}4 \times 10^{10} \\
\left(2 \times 10^{11}\right)^{* *}\end{array}$ & $3 \times 10^{3}$ & $5 \times 10^{-4}$ & \\
\hline & 220 & & 800 & $\begin{array}{c}1.5 \times 10^{-6} \\
\left(1.0 \times 10^{-5}\right)^{*}\end{array}$ & $\begin{array}{c}2 \times 20^{10} \\
\left(1.3 \times 10^{11}\right)^{*} * *\end{array}$ & $2 \times 10^{3}$ & $3 \times 10^{-4}$ & $10^{-9}$ \\
\hline & 250 & 0 & 1000 & $\begin{array}{r}6 \times 10^{-7} \\
\left(5.0 \times 10^{-6}\right. \\
\end{array}$ & $\begin{array}{c}7 \times 10^{9} \\
\left(6 \times 10^{10}\right) * * \\
\end{array}$ & $1 \times 10^{3}$ & $1.4 \times 10^{-4}$ & $10-10$ \\
\hline & 400 & & 1.000 & $\frac{2 \times 10^{-7}}{\left(1.5 \times 10^{-6}\right)^{*}}$ & $\begin{array}{c}2.2 \times 10^{9} \\
\left(2 \times 10^{10}\right)^{* *}\end{array}$ & $3 \times 10^{2}$ & $4 \times 10^{-5}$ & $10^{-10}$ \\
\hline
\end{tabular}

( $)^{*}$ Values by P.O. Pedersen.(9)

( )** Values corresponding to the above.

4. Collision of ion with neutral particles

As we have no exact ground for the estimation of mean collision frequency of ion with neutral particles, except that we know the ratio of mean collision frequencies of electron and ion at the normal pressure, we assumed that the above ratio may hold up to the height of $F_{2}$-layer. The ratio is taken as about $400^{(9)}$

\section{Ionic density}

1. Mathematical relations of electronic and ionic densities

As is easily shown, the following relations governing the densities of electron, negative and positive ions are given, taking attachment and detachment as well as recombination processes into account.

$$
\frac{d n}{d t}=q-\alpha n^{+} n-\beta n+\gamma n^{-}+\delta n^{-},
$$




$$
\begin{aligned}
\frac{d n^{-}}{d t} & =\beta n-\gamma n^{-}-\delta n^{-}-a_{i} n^{+} n^{-}, \\
n^{+} & =n^{-}+n,
\end{aligned}
$$

where

$n, n^{-}, n^{+} \ldots$ number densities $\left(\mathrm{cm}^{-3}\right)$ of electron, negative and positive ions respectively,

$q \ldots \ldots \ldots$ ion production rate $\left(\mathrm{cm}^{-3} \cdot \mathrm{sec}^{-1}\right)$ by solar radiation,

a. .......... recombination coefficient $\left(\mathrm{cm}^{3} \cdot \mathrm{sec}^{-1}\right)$ of electron and positive ion.

$a_{i} \ldots \ldots \ldots$ recombination coefficient $\left(\mathrm{cm}^{3} . \mathrm{sec}^{-1}\right)$ of negative and positive ions,

$\beta$.........attachment coefficient $\left(\mathrm{sec}^{-1}\right)$ of eleciron to neutral particle,

$\gamma$.........photo-detachment coefficient $\left(\mathrm{sec}^{-1}\right)$ of electron from negative ion,

$\delta \ldots \ldots \ldots$ detachment coefficient $\left(\mathrm{sec}^{-1}\right)$ of electron from negative ion by collision.

During the night we may put $q=\gamma=0$.

2. Attachment coefficient $(\beta)$

Concerning $\beta$ for $F$-region, some theoretical results ${ }^{(6)(11)}$ on attachment cross section of Oxygen atom and many experimental data ${ }^{(11)}$ are available, but as for $E$-region, the value is speculative. Considering simply the pressure dependence of $\beta$ we use the values in the above table for our present work.

3. Recombination coefficient ( $\alpha$ and $\mu_{i}$ )

Besides the theoretical formulas, a great many experimental data ${ }^{(12)}$ are available. Considering that the observed value of $\boldsymbol{\alpha}$. is actually the apparent value $\boldsymbol{\alpha}^{\prime}$, which is equal to $(1+\lambda) \boldsymbol{a}\left(\lambda=n^{-} / n\right)$, we must be carefull to utilize the experimental values.

From experiments we may take $\alpha^{\prime}$ as follows.

$$
\begin{array}{ll}
\alpha^{\prime} \simeq 1 \times 10^{-8} \mathrm{~cm}^{3} \mathrm{sec}^{-1} & \text { for } E \text {-region, } \\
\alpha^{\prime} \simeq 1 \times 10^{-10} \mathrm{~cm}^{3} \mathrm{sec}^{-1} \text { for } F \text {-region. }
\end{array}
$$

Concerning $\sigma_{i}$ we have little knowledges, ${ }^{(9)}$ and in the following considerations we touch this quantity. only little, though in the more detailed study $a_{i}$ should be taken into account.

4. Criterion from the increase of electron density of $F_{2}$-layer near the sunrise at ground.

When we consider the rapid increase of $F_{2}$-layer electron density from the sunrise at $F$-layer height $(\mathrm{Ca} .250 \sim 300 \mathrm{~km})$ to near the one at ground, which is very remarkable especially in Winter (from November to February), we can explore rough values of $\gamma+\delta$ (detachment) and then $n^{+}$and $n^{-}$.

As $q$ is zero for the above period of day, we obtain the following equations from equation (2).

$$
\begin{aligned}
\frac{d n}{d t} & =(\gamma+\delta) n^{-}-\alpha n^{+} n-\beta n, \\
& \simeq(\gamma+\delta) n^{+}-(\gamma+\delta+\beta) n,
\end{aligned}
$$

where $a n^{+} n$ is neglected in comparison to $\beta n$. 
If we consider $n^{+}$as constant for the period under consideration, the above equation is solved as follows.

$$
n \simeq \frac{\gamma+\delta}{\beta+\gamma+\delta} n^{+}\left\{1-e^{-(\beta+\gamma+\delta) t}\right\}+n_{0} e^{-(\beta+\gamma+\delta) t},
$$

where $n_{0}$ is the value of $n$ at the sunrise of $F$-region height.

The above equation is rewritten in the following form.

$$
n / n_{0} \simeq \frac{\gamma+\delta}{\beta+\gamma+\delta}(1+\lambda)\left\{1-e^{-(\beta+\gamma+\delta) t}\right\}+e^{-(\beta+\gamma+\delta) \lambda} .
$$

When we use the value $10^{-4}$ as $\beta$, and $3 \times 10^{-3}$ as $\gamma$, which is reported by some theoretists, ${ }^{(9)}$ ( $\delta$ is taken as less than $\beta$ ), we get for $n / n_{0}$ after 333 seconds (about 5.5 min.),

$$
n / n_{0} \simeq 0.63(1+\lambda)+0.37 \text {. }
$$

This value is too much, unless $\lambda$ is taken as very small compared with unity. But with such small $\lambda$ the actual observed curve of $n / n_{0}$ deviates greatly from the equation (7) for the later part of the period. Then it may be supposed that $\gamma$ (including $\delta$ ) should be as low as of the order of $10^{-4}$, but in this case the constancy of $\lambda$ or $n^{+}$ is violated.

In treating the equation $\left(5^{\prime}\right)$, we may proceed on another way as follows. The equation $\left(5^{\prime}\right)$ can be written in the following form,

$$
\frac{d n}{d t}=(\gamma+\delta) \lambda n-\beta n \text {. }
$$

Considering $\lambda$ as variable with time we get the solution,

$$
\frac{n}{n_{0}}=e^{(\gamma+\delta) \int \lambda d t-\beta t},
$$

and for $n^{-}$the solution $\left(n_{0}^{-}\right.$is the initial value of $\left.n^{-}\right)$,

$$
\frac{n^{-}}{n_{0}^{-}}=e^{\beta \int \frac{1}{\lambda} d t-(\gamma+\delta) t} .
$$

When we compare the above equation $\left(5^{\prime \prime}\right)$ or (8) with the actual increase of $n$ in $F_{2}$-layer in Winter by taking $\beta$ as $10^{-4}$, we find that the value of $\gamma+\delta$ as the order of $10^{-4}$ with $\lambda$ of the order of unity will be the most probable, but $n^{+}\left(=n+n^{-}\right)$varies considerably in this case and this is unreasonable for the period under consideration.

Then it may need, so far as the coincidence of the theoretical equations and the actual observed curves is concerned, that both $\gamma+\delta$ and $\lambda$ are considered to vary for the period, subject to constancy on $n^{+}$. In any case the coincidence is more or less insufficient, but it is recommendable that $\gamma$ (including $\delta$ ) should be taken as $10^{-1} \sim 3 \times 10^{-4}$ with $\lambda \simeq 1 \sim 3$.

And in these estimations we find also that $\lambda$ just before the sunrise at $F_{2}$-layer height will be of the order of 3 10, which may ae the night value for $\lambda$.

In these considerations the heights of maximum electron density of $F_{2}$-layer for day and night are taken as 260 and $300 \mathrm{~km}$ respectively and the values of electron density at constant height are used for the calculation.

5. Ionic density of $F_{2}$-region

In the daytime, we get the following relation for around noon, which the 
electronic density rearches near its maximum value.

$$
n=\frac{q+(\gamma+\delta) n^{-}}{\beta+\alpha n^{+}} .
$$

And as a prelimimary approximation we have the relation,

$$
n \simeq \frac{\gamma+\delta}{\beta+\gamma+\delta} n^{+}
$$

Using the values

$$
\text { we get } \quad \begin{aligned}
\beta & =1.0 \times 10^{-4} \quad(\text { for } 260 \mathrm{~km}), \\
& =4 \times 10^{-5} \quad(\text { for } 300 \mathrm{~km}), \\
\gamma+\delta & =10^{-4} \sim 3 \cdot 10^{-4}, \\
n & \simeq \frac{1}{2} n^{+} \sim \frac{3}{4} n^{+} \quad(\text { for } 260 \mathrm{~km}), \\
& \simeq \frac{2}{3} n^{+} \sim n^{+} \quad(\text { for } 300 \mathrm{~km}),
\end{aligned}
$$

and then $\quad n^{+}+n^{-}=3 n \sim \frac{5}{3} n \quad$ (for $260 \mathrm{~km}$ ),

$$
=2 n \sim n: \quad \text { (for } 300 \mathrm{~km}) \text {. }
$$

For the above values the equation (9) holds roughly with the magnitude of $q \simeq 100$.

In the night, when the electronic density decreases to its final value, we get with neglection of $\alpha n^{+} n$,

$$
\lambda=\frac{n^{-}}{n}=\frac{\beta}{\delta} .
$$

Although $\delta$ is not exactly known, $\delta$ must be less than $10^{-4}$ by the consideration of the preceding paragraph, and we assume $n^{-} / n$ as $2 \sim 10$ (the former for $300 \mathrm{~km}$ and the latter for lower than $250 \mathrm{~km}$.) and consequently $n^{+}+n^{-}$as about $5 \sim 20$ times of $n$ during night for $F$-layer,

6. Ionic density of $E$-region

For $E$-region $\beta$ was given in the table. In the daytime we have from various experiments the following relation of electron density $(n)$ around noon, $\left(\frac{d n}{d t}-\right.$ is put to zero)

$$
q \simeq a^{\prime} n^{2}=(1+\lambda) \alpha n^{2} .
$$

As the above relation is fairly accurate, the following relation must hold from the equations (2) and (11):

$$
\begin{aligned}
& \beta n \simeq(\gamma+\delta) n^{-}, \\
\therefore \quad & \frac{n^{-}}{n}=\frac{\beta}{\gamma+\delta} .
\end{aligned}
$$

If we take $\gamma$ as independent of height $\left(10^{-1} \sim 3 \times 10^{-4}\right)$ and $\delta$ as $10^{-3}$, we get following results.

$$
\begin{aligned}
\lambda=\frac{n^{-}}{n} & \simeq 20 \sim 15 \quad(\text { for } 100 \mathrm{~km}), \\
& \simeq 7 \sim 6 \quad(\text { for } 110 \mathrm{~km}) .
\end{aligned}
$$


And for the night, if we neglect $\delta$, we get the following equation from (3), considering $\frac{d n^{-}}{d t}=\gamma=0$,

and then

$$
n / n^{+} n^{-} \simeq \frac{\sigma_{i}}{\beta}
$$

$$
\lambda=\frac{1}{\sqrt{n}} \sqrt{\frac{\beta}{U_{i}}} .
$$

Putting $\sigma_{i} \simeq 10^{-9}$ and $n$ as less than $10^{-1}$, we get

$$
\begin{array}{ll}
\lambda>45 & \text { (for } 100 \mathrm{~km}), \\
\lambda>28 & \text { (for } 110 \mathrm{~km}) .
\end{array}
$$

And when only $\delta$ is taken into account ( $\alpha_{i}$; neglected), $\lambda$ will decrease from the above values to about 20 and 8 respectively, and therefore the above values of $\lambda$ will be the upper limits.

As the consequence we have

$$
\begin{aligned}
& \left.\begin{array}{rl}
n^{+}+n^{-} & \simeq 15 \sim 13, n \text { (average } 14) \quad(\text { for } 110 \mathrm{~km}) ; \\
& \simeq 41 \sim 31, n \text { (average } 36) \quad(\text { for } 100 \mathrm{~km}),
\end{array}\right\} \text { for daytime, } \\
& \left.\begin{array}{l}
\simeq 60 n \text { at most (average } 30 \text { ) (for } 110 \mathrm{~km} \text { ), } \\
\simeq 90 . n \text { at most (average } 60 \text { ) (for } 100 \mathrm{~km} \text { ). }
\end{array}\right\} \text { for night. }
\end{aligned}
$$

\section{Vertical distribution curves}

1. $n$ and $n^{+}+n^{-}$

From the above considerations we reached the stage to draw vertical distribution curves of necessary values. For the distribution of electron density $n$ (lower than the maximum density) we uscd the observational data and for the other part the square root curve of ion production $q$ by Chapman's theory was used. In the figure shown the maximum electron densities of $E$ and $F_{2}$-layers for daytime are taken as $10^{5}$ at $110 \mathrm{~km}$ and $10^{6}$ at $260 \mathrm{~km}$ respectively. From this curve we draw the curve of $n^{+}+n^{-}$, where the height dependency of necessary elements is taken into account, and the curve is shown in the figure.

2. Electrical conductivity $(\sigma)$

The electrical conductivity $\sigma$ of the ionosphere is given by the following equation.

$$
\sigma=\frac{e^{2} n}{m} \frac{\nu}{\omega_{11}^{2}+\nu^{2}}+\frac{e^{2}\left(n^{+}+n^{-}\right)}{M} \frac{\nu_{i}}{\omega_{I i}^{2}+\nu_{i}^{2}} \text { esu, }
$$

where

$\nu_{i} \ldots$...mean collision frequency of ion with neutral particle,

$\omega_{H} \ldots .$. geomagnetic gyrofrequency for electron $\left(\mathrm{sec}^{-1}\right)$,

$\omega_{H i} \ldots \ldots$ geomagnetic gyrofrequency for ion $\left(\mathrm{sec}^{-1}\right)$,

$m, M \ldots \ldots$ mass of electron and ion respectively,

$e$......charge of electron.

In the calculation of $\sigma$ we used the following values. 


$$
\begin{aligned}
M / m & =3 \times 10^{4}, \\
\omega_{I H} & =6.6 \times 10^{6} \quad(H=0.4 \text { Gauss }), \\
\omega_{I i} & =2.3 \times 10^{2} \quad(\quad, \quad, \quad) .
\end{aligned}
$$

The other values necessary for the calculation of $\sigma$ can be seen in the figure and the table. The vertical distribution of $\sigma$ for daytime is calculated and shown in the same figure. As the electronic conductivity is very small in both layers, only $E$-layer daytime conductivity of electron is shown.

According to these curves and some numerical calculations based on the curves the followings can be said. For the daytime the maximum conductivities of $E$ and $F$-layers are $1.7 \times 10^{-14}$ e.m.u. and $1.3 \times 10^{-14}$ e.m.u. respectively and the integrated values for $E$ and $F$-layers are $1.1 \times 10^{-7}$ e.m.u. $(60 \%)$ and $0.7 \times 10^{-7}$ e.m.u. (40\%) respectively, the boundary between the two layers being taken as $190 \mathrm{~km}$. The total is abou $1.8 \times$ $10^{-7}$ e.m.u. The conductivity is rather sensible for the height, and the decrease of $F$-layer height and the increase of $E$-layer height make the conductivity increase.

For the night the total conductivity of $E$-layer decreases roughly to less than one fifth of the daytime value and that of $F$-layer to one eighth, because the increase of height at night decrease $\sigma$ greatly. The over all conductivity is about $3.15 \times 10^{-3}$ e.m.u. at most, i.e. one sixth of the daytime value. The situation (mayimum condition) during night is shown by the dotted curves in the same figure. (The explanation is omitted.)

\section{Concluding remarks}

It is to be noticed that the conductivity during daytime depends upon the ionic density of $E$ and $F$-layers instead of electron density. The contribution of $E$-layer is greater than that of $F$-layer. During night the contribution of $E$-layer is much greater than that of $F$-layer. The diurual change of the conductivity is represented partly by the electron density variation of $E$-layer, to which the ionic density of $E$-layer is closely connected, and partly by the height and electron density variation of $F$-layer.

Although it must be said that some dangerous speculations are involved in this estimation of the electrical conductivity, the study will show an example of the efforts that can be done if one aims to estimate the electrical conductivity directly from the knowledge of ionosphere. We expect further studies on this line and the consideration of calm and disturbed time conditions of ionosphere and terrestrial magnetism.

In conclusion the writer wishes to express his thanks to Dr. T. Nagata and Mr. Yonezawa for their valuable advices. 


\section{References}

(1) T. Nagata and M. Sugiura: Rep. Ionos. Res. Japan (R.I.R.J.), 3, 41-72 (1949).

M. Sugiura : R.I.R.J., 4, 31-36 (1950).

T. Nagata: R.I.R.J., 4, No. 3 (1950) (under press).

G. Ishikawa: R.I.R.J., 4, No. 3 (1950) (under press).

(2) E.V. Appleton: Proc. Roy. Soc. London A, 162, 451-479 (1937).

(3) G. Goubau : H.F.T.E.A., 46, 37-49 (1935).

(4) F.L. Mohler: Journ. Res. National Bureau of St., 25, 507 (1940).

(5) D.R. Bates and H.S.W. Massey: Proc. Gen. Ass. in Stockholm (U.R.S.I.), 7, 299-319 (1949).

(6) T. Yamanouchi : R.I.R.J., 3, 1-15 (1949)

(7) Farmèr and Ratcliffe: Proc. Roy. Soc., 151, 307 (1935).

White and Brown: Proc. Roy. Soc., 153, 645 (1936).

(8) T. Yonezawa: Notes of Res. of P.I.R.W. Japan, No. 3, 1-23 (1948).

(9) P.O. Pedersen: "The Propagation of Radio Waves" (1927).

(10) T. Tukada: Rep. Rad. Res. Japan, 7, No. 2, Oct. (1937).

(11) T. Kono: J.E.C.E. Japan, 28, 233-239 (1944).

F.L. Mohler : loc. cit.

T. Yonezawa: Notes of Res. of P.I.R.W. Japan, No. 1, 11-20 (1947).

T. Tukada: loc. cit.

(12) E.V. Appleton: Rep. on Progress in Phys., 2, 129-165 (1935).

L. Harang: Terr. Mag., 41, 143 (1936) ; 42, 55 (1937).

T. Yonezawa: loc. cit.; Rep. of Synthetic Studies of Joint Obs., No. 2, 43 (1947);

No. 3, p. 18 (1948); R.I.R.J., 4, 79-85 (1950).

K. Maeda and T. Kono: J.E.C.E. Japan, No. 232, 486-495 (1942),

F.L. Mohler : loc. cit.

R. Hechtel : H.F.T.E.A., 56-57, 108-111 (1940). 